\title{
ANÁLISIS COMPARATIVO DE LAS PROPIEDADES FÍSICO-MECÁNICAS DE LA MADERA DE TECA (Tectona grandis L.F.) DE QUEVEDO Y BALZAR
}

\author{
Rommel Crespo Gutiérrez ${ }^{1}$, Edwin Jiménez Romero², Pedro Suatunce Cunuhay ${ }^{1}$, \\ Guillermo Law Blanco ${ }^{2}$ y Carlos Sánchez Fonseca ${ }^{1}$

\begin{abstract}
${ }^{I}$ División Forestal, Unidad de Investigación Cientifica y Tecnológica, Universidad Técnica Estatal de Quevedo. Av. Walter Andrade. Km 1 1⁄2 vía a Santo Domingo, C.P. 73. Quevedo, Los Rios, Ecuador. rscgtexcmex@hotmail.com ${ }^{2}$ Escuela de Ingeniería Forestal, Facultad de Ciencias Ambientales, Universidad Técnica Estatal de Quevedo.
\end{abstract}

\section{RESUMEN}

Se estudió las propiedades físico-mecánicas de la madera de Teca (Tectona grandis L.F.) procedente de Quevedo y Balzar. Los objetivos fueron: determinar el porcentaje de duramen y albura de los árboles, las propiedades físicas en estado verde y anhidro de la madera, las propiedades mecánicas en estado de equilibrio y las principales diferencias físico-mecánicas de la madera. En Quevedo se colectaron cinco árboles de teca de 22 años de edad y en Balzar se colectaron cinco árboles de teca de 18 años de edad. De cada árbol se obtuvieron dos trozas de $2.50 \mathrm{~m}$ de largo y de cada troza se obtuvo un tablón central de $10 \mathrm{~cm}$ de ancho por $2.50 \mathrm{~m}$ de longitud del cual se extrajo las viguetas y finalmente las probetas. Se elaboraron 60 probetas (seis probetas por árbol) para los ensayos de las propiedades físicas. Para el estudio de las propiedades mecánicas se elaboraron 60 probetas (una por cada ensayo por árbol). La metodología empleada para el estudio de las propiedades físicas y mecánicas se fundamentó en las normas ASTM D 143-94 (American Society for Testing and Materials). Los resultaron mostraron que no existe diferencia significativa entre la madera de las dos zonas. La madera de Teca de Quevedo y Balzar presentó mediana densidad, por lo que tiene mejores posibilidades de uso en la fabricación de muebles y gabinetes, usos exteriores, molduras, parquet y pisos en general, carpintería en general, entablados, puertas y ventanas, herramientas agrícolas y vehículos, entre las principales.

Palabras clave: Propiedades físico-mecánicas, Tectona grandis L.F.

\section{ABSTRACT}

It was studied the physical-mechanical properties of the wood Teak (Tectona grandis L.F.) coming from Quevedo and Balzar. The objectives were: to determine the heartwood and sapwood percentage of the trees, the physical properties in green and anhydrous state of the wood, the mechanical properties in balance state and the main physical-mechanical differences of the wood. In Quevedo five trees of 22 years old teak were collected and in Balzar five trees of 18 years old teak were collected. Of each tree two logs of $2.50 \mathrm{~m}$ of long was obtained and of each $\log$ a central plank of $10 \mathrm{~cm}$ of wide by $2.50 \mathrm{~m}$ of longitude was obtained of which beams was extracted and finally the specimens. 60 specimens were elaborated (six specimens for tree) for the rehearsals of the physical properties. For the study of the mechanical properties 60 specimens were elaborated (one for each rehearsal for tree). The methodology used for the study of the physical and mechanical properties was based in the norms ASTM D 143-94 (American Society for Testing and Materials). The results showed that significant difference doesn't exist among the wood of the two areas. The wood of Teak of Quevedo and Balzar presented medium density, for what has better use possibilities in the production of furniture and cabinets, external uses, molds, parquet and floors in general, carpentry in general, boardings, doors and windows, agricultural tools and vehicles, among the main ones.

Key words: Physical-mechanical properties, Tectona grandis L.F.

\section{INTRODUCCIÓN}

La Teca es una especie latifoliada que pertenece a la familia Verbenaceae. Es un árbol grande, deciduo, que puede alcanzar más de $50 \mathrm{~m}$ de altura y $2 \mathrm{~m}$ de diámetro en su lugar de origen. Es un árbol de fuste recto, con corteza áspera y fisurada de $1.2 \mathrm{~mm}$ de espesor, de color café claro que desfolia en placas grandes y delgadas (Fonseca, 2004). La distribución natural abarca de los 10 a los $25^{\circ} \mathrm{N}$ en el Subcontinente Indio y el Asia Suroriental, especialmente en India, Myanmar, Tailandia, Laos Camboya, Vietnam y en Java. En Malasia no es nativa (Lamprecht, 1990 citado por Rivero, 2004). En América Tropical fue introducida primero en

Recibido: Octubre, 2007. Aceptado: Febrero, 2008. Publicado como ARTÍCULO en Ciencia y Tecnología 1: 55-63. 2008.
Trinidad en 1913 y en 1916, con semillas procedentes de Tenasserim en Burma (Myanmar). Esta procedencia ha sido ampliamente distribuida, exportándose semilla de Trinidad a Belice, Antigua, Dominicana, Jamaica, Costa Rica, Cuba, Colombia, Venezuela, Haití, Puerto Rico, Ecuador, Guayana Francesa y México (Fonseca, 2004).

Esta madera es una de las más versátiles, valiosas, de gran valor y prestigio. Además de su apariencia es distintivo su color amarillo bronceado a café oscuro, a menudo con líneas acentuadas claras y oscuras. Es, talvez, la madera más vistosa en cortes transversales. Tiene propiedades únicas de estabilidad, haciéndola ideal para aplicaciones exteriores. Su elevado costo es usualmente el limitante para utilizarla en trabajos de 
decoración interior. Tiene una gran belleza y se la prefiere con acabado "al natural" (AWI, 1997 citado por Rivero, 2004).

Intrínsecamente, la Teca es una de las maderas más valiosas, pero su uso está limitado por su escasez y alto costo. Es única en cuanto no causa herrumbre o corrosión cuando está en contacto con metales; por lo cual, es muy usada en la industria de construcción naval, tanques y toneles, y otros que requieran alta resistencia a los ácidos. Actualmente utilizada en la construcción de costosos botes, muebles, pisos, objetos decorativos y exteriores decorativos en tableros aglomerados (USDA, 1999 citada por Rivero, 2004).

Los ensayos de las propiedades físicas y mecánicas de las maderas se realizan sea cual sea la norma utilizada, generalmente con el objeto de proponer los usos probables y dar al ingeniero los datos necesarios para el cálculo de estructuras de maderas. Es necesario que los datos obtenidos permitan el uso adecuado de la madera en proporción mínima, y que garanticen seguridad en cuanto a parámetros de diseño (Hoheisel, 1981 citado por Encalada y Castillo, 1988).

El comportamiento físico de la madera está constituido por una serie de propiedades tales como contenido de humedad, densidad, peso específico, etc., las cuales en conjunto pueden definirse como propiedades físicas de la madera. Partiendo de esta concepción, las propiedades físicas de la madera son el conjunto de propiedades que caracterizan el comportamiento físico de la misma (Arroyo, 1983 citado por Rivero, 2004).

En resumen se puede decir que la selección y utilización de la madera de una determinada especie con fines industriales, sólo podrán ser realizadas con el conocimiento preciso de las cualidades tanto físicas como mecánicas de la madera (Serrano et al, 2002 citados por Rivero, 2004).

Las propiedades mecánicas de la madera son la expresión de su comportamiento bajo la aplicación de fuerzas o cargas. Este comportamiento puede sufrir varias modificaciones, dependiendo del tipo de fuerza aplicada y de las diferencias básicas en la organización estructural de la madera (Pérez, 1983 citado por Encalada y Castillo, 1988). El conocimiento de las propiedades mecánicas de la madera proporciona los datos básicos requeridos por la ingeniería en el cálculo y diseño para la construcción y otros usos (Pérez y Alcántara, 1983 citados por Jaramillo y Ontaneda, 1989).

\section{MATERIALES Y MÉTODOS}

La investigación se realizó en la zona de Quevedo se encuentra a $73 \mathrm{msnm}$, presenta una precipitación anual de 2,355.38 $\mathrm{mm}$, una temperatura promedio anual de $25.58{ }^{\circ} \mathrm{C}$, una humedad relativa de $85.93 \%$, una heliofanía media anual de 879.42 horas luz y un tipo de suelo franco limoso con un $\mathrm{pH}$ de 6.5-7.0. La zona de Balzar se encuentra a $40 \mathrm{msnm}$, presenta una precipitación media anual de $1,222 \mathrm{~mm}$, una temperatura promedio anual de $24.4{ }^{\circ} \mathrm{C}$, una humedad relativa de $72.9 \%$, una heliofanía media anual de 881 horas luz y un tipo de suelo arcilloso-arenoso con un pH 5.9-7.

Se eligieron al azar 5 árboles en Quevedo y 5 árboles en Balzar, los cuales fueron apeados utilizando una motosierra. Estos árboles presentaron buenas características fitosanitarias, fustes rectos, cilíndricos y fueron representativos tanto en diámetro como en altura. Los datos de los árboles en pie y apeados fueron registrados en una hoja de campo.

El cálculo de la cantidad aproximada de duramen y albura existentes en cada una de las trozas, de las cuales se extrajo las probetas para los diferentes ensayos, se efectuó mediante una relación entre el volumen total y el volumen de duramen, aplicando las siguientes fórmulas:

$$
\begin{gathered}
\text { Volumen total }\left(\mathrm{m}^{3}\right)=0.7854 * \text { largo } *\left(\frac{\text { Diámetro mayor }+ \text { diámetro menor }}{2}\right)^{2} \\
\text { Volumen albura }=\text { volumen total }- \text { volumen duramen } \\
\text { Albura\% }=\frac{\text { volumen albura } * 100}{\text { volumen total }} \\
\text { Duramen } \%=100-\text { albura } \%
\end{gathered}
$$

Una vez apeado se extrajeron dos trozas de cada árbol y de cada una de ellas se obtuvo un tablón central de $10 \mathrm{~cm}$ de ancho por $2.50 \mathrm{~m}$ de largo, los cuales fueron marcados para su posterior identificación.

De cada tablón se obtuvieron viguetas de $10 \times 10$ de ancho por $250 \mathrm{~cm}$ de largo. De una de estas viguetas utilizando canteadora, sierra circular, cepilladora y lijadora se elaboraron 60 probetas para el ensayo de las propiedades físicas de acuerdo a las normas ASTM (1996) y teniendo en cuenta la correcta orientación de las caras. Puesto que los árboles fueron apeados varias semanas antes, las probetas fueron sumergidas en agua durante 24 horas para que adquirieran suficiente cantidad de agua y que el contenido de humedad sea mayor al 30\%. En cada una de las probetas se determinó su peso, volumen y dimensiones en el estado verde y seco al horno, para lo cual se utilizó una balanza digital, un calibrador y una estufa eléctrica.

Las propiedades físicas del contenido de humedad en estado verde y anhidro, densidad, peso específico básico y contracción fueron determinadas por medio del secado en estufa eléctrica (diferencia de peso), provista de un termorregulador en el Laboratorio de Biotecnología 
de la Universidad Técnica Estatal de Quevedo. Se aplicó un calentamiento gradual $\left(45^{\circ} \mathrm{C}, 75^{\circ} \mathrm{C}\right.$ y $\left.103 \pm 2{ }^{\circ} \mathrm{C}\right)$ hasta que las probetas alcanzaron un peso constante. Se utilizaron las siguientes fórmulas:

$$
\begin{gathered}
\text { Contenido humedad verde }(\%)=\frac{\text { Peso húmedo }- \text { Peso seco }}{\text { Peso seco }} \times 100 \\
\text { Contenido humedad anhidro }(\%)=\frac{\text { Peso húmedo }- \text { Peso seco }}{\text { Peso húmedo }} \times 100 \\
\text { Densidad verde }\left(\mathrm{g} \mathrm{cm}^{3}\right)=\frac{\text { Peso verde }}{\text { Volumen verde }} \\
\text { Densidad anhidra }\left(\mathrm{g} \mathrm{cm}^{3}\right)=\frac{\text { Peso seco al horno }}{\text { Volumen seco al horno }} \\
\text { Contracción tangencial total }(\%)=\frac{\text { Peso seco }- \text { Dta }}{\text { Volumen húmedo }} \times 100
\end{gathered}
$$

Donde:

$D t v=$ dimensión tangencial en estado verde

$D t a=$ dimensión tangencial en estado anhidro

$$
\text { Contracción radial total }(\%)=\frac{D r v-D r a}{D r v} \times 100
$$

Donde:

$D r v=$ dimensión radial en estado verde

Dra $=$ dimensión radial en estado anhidro

$$
\text { Contracción longitudinal total }(\%)=\frac{D l v-D l a}{D l v} \times 100
$$

Donde:

$D l v=$ dimensión longitudinal en estado verde

$D l a=$ dimensión longitudinal en estado anhidro

$$
\text { Contracción volumétrica total }=C t t+C r t+C l t
$$

Donde:

$C t t=$ Contracción tangencial total

$C r t=$ Contracción radial total

$C l t=$ Contracción longitudinal total

$$
\text { Relación }{ }^{T} / R=\frac{\text { Contracción Tangencial (\%) }}{\text { Contracción radial (\%) }}
$$

Las 60 probetas para el ensayo de las propiedades mecánicas (1 probeta por árbol, 10 por ensayo) fueron elaboradas de las viguetas, con el contenido de humedad en estado de equilibrio, en el taller de la madera del Laboratorio de Ensayos de Materiales y Modelos de la
Universidad Central del Ecuador en la ciudad de Quito, de acuerdo con las normas ASTM (1996), y fueron sometidas a los ensayos de: flexión estática, compresión paralela y perpendicular a la fibra, dureza Brinnell de extremos y lados, corte paralelo a la fibra y extracción de clavos en las tres caras. Para ello se utilizó la maquina universal de ensayos Tinius Olsen con sus respectivos accesorios. Se realizaron los siguientes cálculos:

\section{Flexión estática}

$$
\begin{gathered}
\begin{array}{c}
\text { Módulo } \\
\text { de ruptura }\left(\mathrm{kg} \mathrm{cm}^{2}\right)
\end{array}=\frac{1.5 \times \text { carga máxima } \times \text { luz entre apoyos }}{\text { Ancho de probeta } x(\text { altura probeta })^{2}} \\
\begin{array}{c}
\text { Módulo de } \\
\text { elasticidad }\left(\mathrm{kg} \mathrm{cm}^{2}\right)
\end{array}=\frac{0.25 \times(\text { luz entre apoyos })^{3} \times C L P}{\text { Ancho de probeta } x \text { (altura probeta) })^{3} \times D L P}
\end{gathered}
$$

Donde:

$C L P=$ Carga en el límite proporcional $(\mathrm{kg})$

$D L P=$ Deformación en el límite proporcional $(\mathrm{cm})$

\section{Compresión paralela a la fibra}

$$
\underset{\text { Módulo }}{\text { de ruptura }\left(\mathrm{kg} \mathrm{cm}^{2}\right)}=\frac{\text { Carga máxima }}{\text { Ancho de probeta x espesor de probeta }}
$$

\section{Compresión perpendicular a la fibra}

$E L P\left(\mathrm{~kg} \mathrm{~cm}^{2}\right)=\frac{\text { Carga en el límite proporcional }}{\text { Ancho de pieza metálica x espesor de pieza metálica }}$

Donde:

$$
E L P=\text { Esfuerzo en el límite proporcional }
$$

\section{Corte paralelo a la fibra}

Esfuerzo máximo $\left(\mathrm{kg} \mathrm{cm}^{2}\right)=\frac{\text { Carga máxima }(\mathrm{kg})}{5 \mathrm{~cm} \times 5 \mathrm{~cm}}$

Los datos para los ensayos de Dureza Brinnell y de Extracción de clavos se obtuvieron directamente de la máquina, sin necesidad de hacer ningún cálculo adicional. Se realizó un análisis de comparación de medias a través de la prueba de " $t$ " a un nivel de significancia de 0.10 .

\section{RESULTADOS}

\section{Porcentaje de duramen y albura}

Las medias porcentuales de duramen y albura de las 10 trozas de teca procedente de Quevedo y 10 trozas de teca procedente de Balzar presentaron diferencias significativas (Cuadro 1).

\section{Propiedades físicas}

El contenido de humedad en estado anhidro, densidad en estado anhidro, peso específico básico, con 
tracción total tangencial y contracción total longitudinal presentaron diferencias significativas; las demás variables no presentaron diferencias significativas (Cuadro 2).

La madera de teca de Quevedo y Balzar se clasificó como de mediana densidad en estado anhidro, bajo peso específico para la teca de Quevedo y medio peso específico para la teca de Balzar, muy baja contracción volumétrica total y de una relación Tangencial/Radial (T/R) alta o inestable. (Cuadro 3).

\section{Propiedades mecánicas}

El módulo de la elasticidad (MOE) en la flexión estática y el módulo de ruptura (MOR) en el corte paralelo a la fibra en la cara radial presentaron diferencias significativas (Cuadro 4).
El MOR para la madera de teca de Quevedo y Balzar en la flexión estática fue bajo. El MOE en la madera de teca de Quevedo fue mediano y bajo en la de Balzar. El MOR en la compresión paralela a la fibra fue bajo Quevedo y muy baja para de Balzar. El ELP en la compresión perpendicular a la fibra también fue bajo para las maderas de las dos zonas. La madera de teca de las dos zonas, en la cara transversal, se clasificó como baja o blanda, de acuerdo a la dureza Brinnell y moderadamente dura en las caras radial y tangencial. El MOR en el corte paralelo a la fibra en la cara radial fue mediano. La madera de teca de ambas zonas presentó una resistencia a la extracción de clavos alta en la cara transversal y muy alta en las caras radial y tangencial (Cuadro 5).

Cuadro 1. Porcentajes de duramen y albura en trozas de teca procedente de las zonas de Balzar y Quevedo

\begin{tabular}{cccc}
\hline \multicolumn{2}{c}{ Duramen $(\%)$} & \multicolumn{2}{c}{ Albura (\%) } \\
\hline Balzar & Quevedo & Balzar & Quevedo \\
\hline $71.89 \mathrm{a}^{\dagger}$ & $59.27 \mathrm{~b}$ & $28.11 \mathrm{~b}$ & $40.73 \mathrm{a}$ \\
\hline
\end{tabular}

Cuadro 2. Valores promedios de las propiedades físicas de la madera de Teca procedente de Quevedo y Balzar

\begin{tabular}{lcc}
\hline \multirow{2}{*}{\multicolumn{1}{c}{ Ensayos }} & \multicolumn{2}{c}{ Localidad } \\
\cline { 2 - 3 } & Quevedo & Balzar \\
\hline Contenido de humedad verde (\%) & $107.09 \mathrm{a}^{\dagger}$ & $93.46 \mathrm{a}$ \\
Contenido de humedad anhidra (\%) & $51.53 \mathrm{a}$ & $47.99 \mathrm{~b}$ \\
Densidad estado verde $\left(\mathrm{g} \mathrm{cm}^{3}\right)$ & $0.98 \mathrm{a}$ & $0.99 \mathrm{a}$ \\
Densidad estado anhidro $\left(\mathrm{g} \mathrm{cm}^{3}\right)$ & $0.52 \mathrm{~b}$ & $0.55 \mathrm{a}$ \\
Peso específico básico & $0.48 \mathrm{~b}$ & $0.52 \mathrm{a}$ \\
Contracción total radial (\%) & $2.04 \mathrm{a}$ & $2.06 \mathrm{a}$ \\
Contracción total tangencial (\%) & $5.66 \mathrm{a}$ & $5.22 \mathrm{~b}$ \\
Contracción total longitudinal (\%) & $0.41 \mathrm{a}$ & $0.49 \mathrm{~b}$ \\
Contracción total volumétrica (\%) & $8.11 \mathrm{a}$ & $7.77 \mathrm{a}$ \\
Relación tangencial/radial & $2.96 \mathrm{a}$ & $2.63 \mathrm{a}$ \\
\hline \$Valor adimensional. &
\end{tabular}


Cuadro 3. Categorización de la madera de teca para la zona de Quevedo y Balzar según los resultados obtenidos en los ensayos de las propiedades físicas

\begin{tabular}{|c|c|c|}
\hline \multirow{3}{*}{ Propiedades } & \multicolumn{2}{|c|}{ Localidad } \\
\hline & Quevedo & Balzar \\
\hline & \multicolumn{2}{|c|}{ Clasificación } \\
\hline Densidad en estado anhidra $\left(\mathrm{g} \mathrm{cm}^{3}\right)$ & Mediana & Mediana \\
\hline Peso específico básico & Bajo & Medio \\
\hline Contracción volumétrica total (\%) & Muy baja & Muy baja \\
\hline Relación contracción tangencial/radial (T/R) & Alta o inestable & Alta o inestable \\
\hline
\end{tabular}

Cuadro 4. Valores promedios de las propiedades mecánicas de la madera de Teca procedente de Quevedo y Balzar

\begin{tabular}{|c|c|c|}
\hline \multirow{2}{*}{ Propiedades } & \multicolumn{2}{|c|}{ Localidad } \\
\hline & Quevedo & Balzar \\
\hline \multicolumn{3}{|l|}{ Flexión Estática } \\
\hline Módulo de ruptura (MOR, $\mathrm{kg} \mathrm{cm²)}$ & $830.49 \mathrm{a}^{\dagger}$ & $755.48 \mathrm{a}$ \\
\hline Módulo de elasticidad (MOE, $\mathrm{kg} \mathrm{cm}{ }^{2}$ ) & $106,553.49 \mathrm{a}$ & $93,449.65 \mathrm{~b}$ \\
\hline \multicolumn{3}{|l|}{ Compresión paralela a la Fibra } \\
\hline Módulo de ruptura (MOR, kg cm²) & $426.91 \mathrm{a}$ & $388.18 \mathrm{a}$ \\
\hline \multicolumn{3}{|l|}{ Compresión perpendicular a la fibra } \\
\hline Esfuerzo al límite proporcional (ELP, $\left.\mathrm{kg} \mathrm{cm}^{2}\right)$ & $59.20 \mathrm{a}$ & $58.43 \mathrm{a}$ \\
\hline \multicolumn{3}{|l|}{ Corte paralelo a la fibra cara radial } \\
\hline Módulo de ruptura (MOR, kg cm²) & $89.45 \mathrm{~b}$ & $106.33 \mathrm{a}$ \\
\hline \multicolumn{3}{|l|}{ Extracción de clavos } \\
\hline Cara radial (carga máxima, kg) & $88.70 \mathrm{a}$ & $74.00 \mathrm{a}$ \\
\hline Cara tangencial (carga máxima, kg) & $86.50 \mathrm{a}$ & $77.50 \mathrm{a}$ \\
\hline Cara Transversal (carga máxima, kg) & $60.00 \mathrm{a}$ & $58.00 \mathrm{a}$ \\
\hline \multicolumn{3}{|l|}{ Dureza Brinnell } \\
\hline Cara radial (carga máxima, kg) & $449.50 \mathrm{a}$ & $425.50 \mathrm{a}$ \\
\hline Cara tangencial (carga máxima, kg) & $423.50 \mathrm{a}$ & $426.00 \mathrm{a}$ \\
\hline Cara transversal (carga máxima, kg) & $383.50 \mathrm{a}$ & $423.50 \mathrm{a}$ \\
\hline
\end{tabular}




Cuadro 5. Categorización de la madera de teca para la zona de Quevedo y Balzar según los
resultados obtenidos en los ensayos de las propiedades mecánicas

\section{DISCUSION}

\section{Porcentaje de duramen y albura}

Los porcentajes de duramen de la teca de Quevedo y Balzar, encontrados en esta investigación, difieren con los expresados por Fonseca (2004) quien reporta que la madera de teca presenta una proporción de duramen de $55 \%$ a los 30 años, aumentando logarítmicamente conforme avanza la edad y aumenta el diámetro. Además afirma que valores de 33 y $37 \%$ de duramen fueron encontrados en árboles de teca de 10 años de edad en la región seca de Costa Rica y que similares valores se obtuvieron en la región húmeda para árboles de la misma edad.

\section{Propiedades físicas}

El valor de $93.46 \%$ para el contenido de humedad en estado verde determinado en la madera de Balzar de 18 años, concuerda con el determinado por Castro y Raigosa (2000) en Costa Rica, quienes para madera de teca de 17 años reportaron un valor de 93\%. El valor del peso específico de 0.52 calculado en la madera de teca de Balzar se parece al encontrado por Córdoba y Sáenz (2004) en teca de 20 años en Costa Rica quienes determinaron un valor de 0.53 y 0.57 . Estos mismos investigadores en tecas de 18 a 20 años en Panamá determinaron un peso específico de 0.63 , el cual difiere con el encontrado en Balzar. En cambio el resultado de Balzar se parece al encontrado por Castro y Raigosa (2000) de 0.58 en árboles de 17 años en Costa Rica.

Los valores de densidad verde de $0.98 \mathrm{~g} \mathrm{~cm}^{3}$ para la teca de Quevedo de 22 años y de $0.99 \mathrm{~g} \mathrm{~cm}^{3}$ para la teca de Balzar de 18 años, no se parecen a los encontrados por Córdoba y Sáenz (2004) en Costa Rica en teca de 20 años con $0.82 \mathrm{~g} \mathrm{~cm}^{3}$ y $0.86 \mathrm{~g} \mathrm{~cm}^{3}$, y en Panamá en teca de 18 a 20 años un valor de $0.94 \mathrm{~g} \mathrm{~cm}^{3}$ el cual se asemeja a los encontrados en Quevedo y Balzar. La contracción volumétrica total de $8.11 \%$ para la teca de Quevedo y de $7.77 \%$ para la teca de Balzar no concuerda con los valores encontrados por Córdoba y Sáenz (2004) en tecas de 20 años en Costa Rica, quienes reportaron un valor de $6.55 \%$ y en teca de 25 años un valor de $6.09 \%$.

La relación de contracción Tangencial/Radial $(\mathrm{T} / \mathrm{R})$ de $2.96 \%$ para la teca de Quevedo y de $2.63 \%$ para la teca de Balzar difieren de los encontrados por Córdoba y Sáenz (2004) en Panamá en teca de 18 a 20 años, los cuales obtuvieron un valor de 1.97, y para teca de 23 a 25 años un valor de $2.25 \%$. La alta relación de contracción tangencial/radial (T/R) en la madera de teca de ambas zonas sugiere que esta madera es relativamente difícil de secar.

\section{Propiedades mecánicas}

Los valores de $830.48 \mathrm{~kg} \mathrm{~cm}^{2}$ y de $755.48 \mathrm{~kg} \mathrm{~cm}^{2}$ para el módulo de ruptura (MOR) y de $106,553.49 \mathrm{~kg}$ $\mathrm{cm}^{2}$ y de $93,449.65 \mathrm{~kg} \mathrm{~cm}^{2}$ para el módulo de elasticidad (MOE) determinados en el ensayo de Flexión Estática para la madera de teca de las zonas de Quevedo y Balzar, respectivamente, difieren con los resultados obtenidos por Govaere et al. (2003) en Costa Rica en teca de 17 años de edad, donde el MOR fue de $991 \mathrm{~kg} \mathrm{~cm}^{2}$ y el MOE de $112,000 \mathrm{~kg} \mathrm{~cm}^{2}$. Difieren 
también con los presentados por Córdoba y Sáenz (2004) en Costa Rica en teca de 20 años, quienes obtuvieron un MOR de $935.87 \mathrm{~kg} \mathrm{~cm}^{2}$ y un MOE $115,404.25 \mathrm{~kg} \mathrm{~cm}^{2}$. Mientras en Panamá en teca de 18 y 20 años de edad obtuvieron un MOR de $755.72 \mathrm{~kg} \mathrm{~cm}^{2}$ y un MOE de $110,402.39 \mathrm{~kg} \mathrm{~cm}^{2}$.

Los valores del esfuerzo al límite proporcional (ELP) obtenidos en el ensayo de compresión perpendicular a la fibra en la madera de teca de la zona de Quevedo de $59.20 \mathrm{~kg} \mathrm{~cm}^{2}$ y de la zona de Balzar de $58.43 \mathrm{~kg} \mathrm{~cm}^{2}$ difieren con los obtenidos por Govaere et al. (2003) en Costa Rica, quienes en teca de 28 años obtuvieron un valor de $85 \mathrm{~kg} \mathrm{~cm}^{2}$ y en teca de 17 años obtuvieron un valor de $98 \mathrm{~kg} \mathrm{~cm}^{2}$.

El valor del módulo de ruptura (MOR) obtenido en el ensayo de corte paralelo a la fibra en la cara radial de la madera de teca de la zona de Quevedo de $89.45 \mathrm{~kg}$ $\mathrm{cm}^{2}$ y de la zona de Balzar de $106.33 \mathrm{~kg} \mathrm{~cm}^{2}$, difieren con los determinados por Córdoba y Sáenz (2004) en teca de 20 años de edad en Costa Rica, quienes obtuvieron un valor de $169.44 \mathrm{~kg} \mathrm{~cm}^{2}$ y en tecas de 25 años un valor de $149.11 \mathrm{~kg} \mathrm{~cm}^{2}$. En Panamá en teca de 18 a 20 años obtuvieron un valor de $200.88 \mathrm{~kg} \mathrm{~cm}^{2}$, en teca de 23 a 25 años un valor de $171.78 \mathrm{~kg} \mathrm{~cm}^{2}$.

La carga máxima determinada en el ensayo de dureza transversal en la teca de Quevedo fue de $383.50 \mathrm{~kg}$ y para la teca de Balzar de $423.50 \mathrm{~kg}$. Estos resultados no concuerdan con aquellos reportados en Costa Rica por Córdoba y Sáenz (2004) quienes en teca de 20 años de edad obtuvieron un valor de $606.25 \mathrm{~kg}$ y en teca de 25 años un valor de $563.64 \mathrm{~kg}$.

\section{CONCLUSIONES}

El Porcentaje de albura en la madera de teca procedente de Quevedo es mayor al determinado en la de Balzar, en cambio, el porcentaje de duramen es mayor en la zona de Balzar.

El mayor contenido de humedad, en estado seco al horno, fue para la teca de la zona de Quevedo. La densidad de la madera de teca, en estado seco al horno fue mayor en Balzar.

El peso específico básico de la madera de teca fue mayor para la teca de Balzar. La contracción radial total y longitudinal total de la madera de teca fueron mayores en la teca de la zona de Balzar. La contracción tangencial total y volumétrica total fueron mayores en la teca de Quevedo. La relación tangencial/radial (T/R) son considerados altos o inestables.

El módulo de elasticidad (MOE) en la flexión estática fue mayor en la zona de Quevedo. El módulo de ruptura (MOR) en el corte paralelo a la fibra, en la cara radial, fue mayor en la zona de Balzar.

En general, la madera de teca de Balzar presentó mejores propiedades físicas, en cambio la madera de teca de Quevedo presentó mejores propiedades mecánicas, por cuanto las condiciones ambientales son diferentes.

\section{LITERATURA CITADA}

ASTM (American Society for Testing and Materials). 1996 Annual Book of ASTM Andards. Section 4 Construction. Volume 04.10 Wood. Pennsylvania, United States. P. 23-39, 556-559.

Castro, F. y Raigosa, J. 2000. Crecimiento y Propiedades Físico-Mecánicas de la Madera de Teca (Tectona grandis) de 17 años de edad en San Joaquín de Abangares, Costa Rica. (En línea). Cr. Propiedades Mecánicas. Consultado el 9 dic. 2005. Disponible en http://www.mag.go.cr/rev_agr/v24n02_007. pdf.

Córdoba, R. y Sáenz, M. 2004. Propiedades FísicoMecánicas de Teca de Costa Rica y Panamá. (En línea). Cr. Introducción. Consultado el 9 dic. 2005. Disponible en www.FundatecITCR.com.

Encalada, O. y Castillo, F. 1988. Propiedades físicas y mecánicas de diez especies forestales de la reserva forestal "La Zarza" en la Provincia de Zamora Chinchipe. Tesis Ing. For. Loja, Ecuador. Universidad Nacional de Loja. Facultad de Ciencias Agrícolas. Escuela de Ingeniería Forestal. 132p.

Fonseca, W. 2004. Manual para productores de Teca (Tectona grandis 1. f) en Costa Rica. (En línea). Cr. Características y Propiedades de la madera. Consultado el 27 jul. 2004. Disponible en http://www. fonafifo.com/text_files/proyectos/manualProdu ctoresTeca.pdf

Govaere, G., Carpio I. y Cruz L. 2003. Descripción anatómica, durabilidad y propiedades físicas y mecánicas de Tectona grandis. (En línea). Cr. Propiedades mecánicas. Consultado el 17 de nov. 2003. Disponible en http://www.una.ac.cr/inis/ docs/teca/temas/ARTICULO\% 20LPF\%201.pdf.

Jaramillo, F., Ontaneda, G. 1989. Estudio de las características tecnológicas, trabajabilidad y usos de la especie forestal Pollalesta karstenii, Pigüe. Tesis. Ing. For. Loja, Ecuador. Universidad Nacional de Loja. Facultad de Ciencias Agrícolas. Escuela de Ingeniería Forestal. 142p.

Rivero, J. 2004 Propiedades Físico-Mecánicas de Gmelina arbórea Roxb. y Tectona grandis Linn. F. (en línea). Bo. Metodología. Consultado el 28 jun del 2005 Disponible en http://www.monografias.com/trabajos16/gmelina-arborea/gmelina-arborea.shtml\#top 
Anexo 1. Normas ASTM empleadas para la fabricación de las probetas utilizadas en los ensayos de las Propiedades Físicas.

\begin{tabular}{llc}
\hline \multicolumn{1}{c}{ Ensayo } & Dimensión $(\mathrm{cm})$ & Norma \\
\hline Densidad & $2.5 \times 2.5 \times 10.0$ & ASTM D-143-94 \\
Peso específico & $2.5 \times 2.5 \times 10.0$ & ASTM D-143-94 \\
Contenido de humedad & $2.5 \times 2.5 \times 10.0$ & ASTM D-143-94 \\
Contracción radial y tangencial & $2.5 \times 2.5 \times 10.0$ & ASTM D-143-94 \\
Contracción longitudinal & $2.5 \times 2.5 \times 10.0$ & ASTM D-143-94 \\
Contracción volumétrica & $2.5 \times 2.5 \times 10.0$ & ASTM D-143-94 \\
\hline
\end{tabular}

Anexo 2. Normas ASTM empleadas para la fabricación de las probetas utilizadas en los ensayos de las Propiedades Mecánicas.

\begin{tabular}{lcc}
\hline \multicolumn{1}{c}{ Ensayo } & Dimensión $(\mathrm{cm})$ & Norma \\
\hline Flexión estática & $5.0 \times 5.0 \times 76.0$ & ASTM D-143-94 \\
Compresión paralela & $5.0 \times 5.0 \times 20.0$ & ASTM D-143-94 \\
Compresión perpendicular & $5.0 \times 5.0 \times 15.0$ & ASTM D-143-94 \\
Dureza Brinnell & $5.0 \times 5.0 \times 15.0$ & ASTM D-143-94 \\
Cizallamiento & $5.0 \times 5.0 \times 6.30$ & ASTM D-143-94 \\
Extracción de clavos & $5.0 \times 5.0 \times 15.0$ & ASTM D-143-94 \\
\hline
\end{tabular}

Anexo 3. Figuras de las probetas y accesorios utilizados en los ensayos de las propiedades físico-mecánicas de la madera de teca.
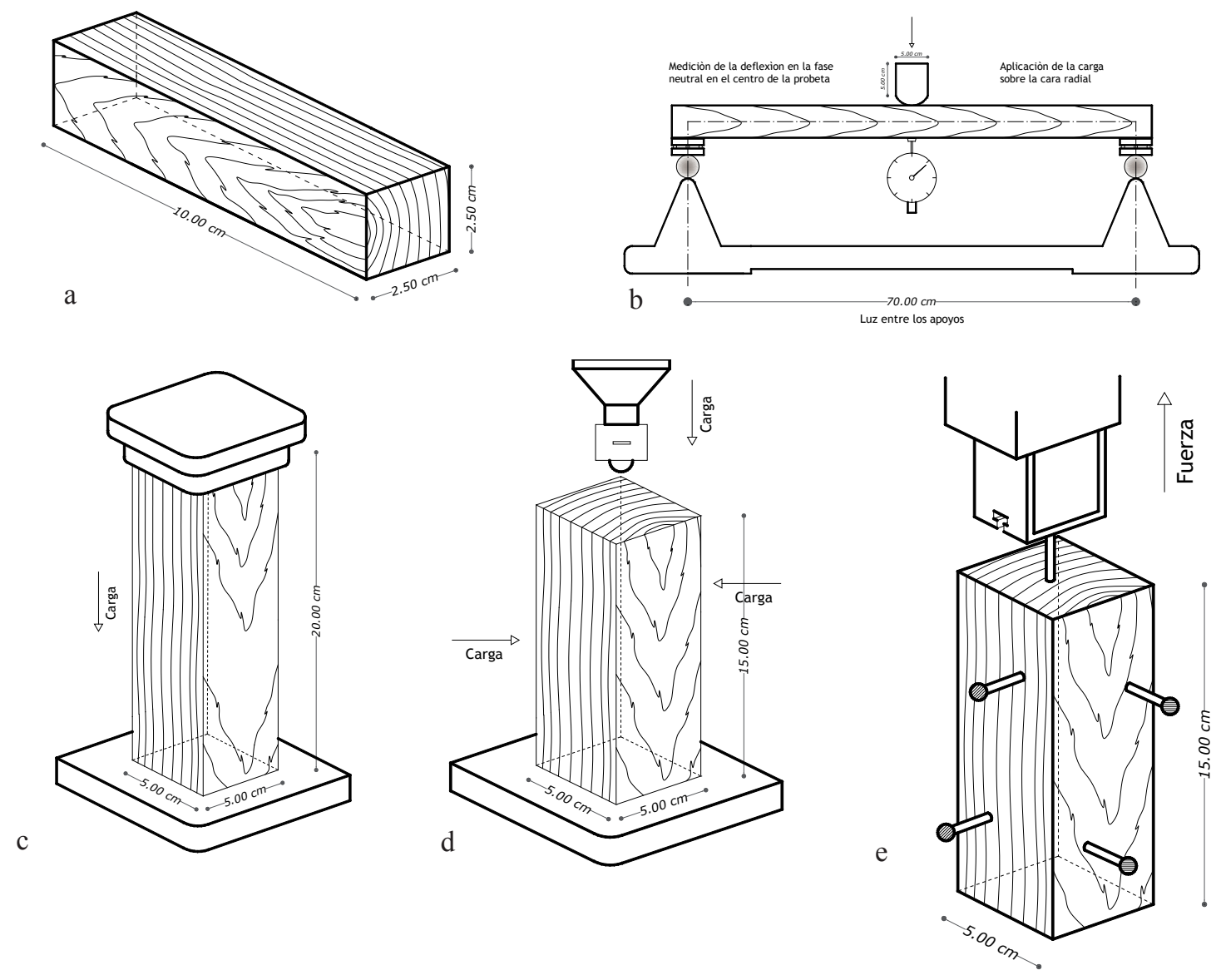

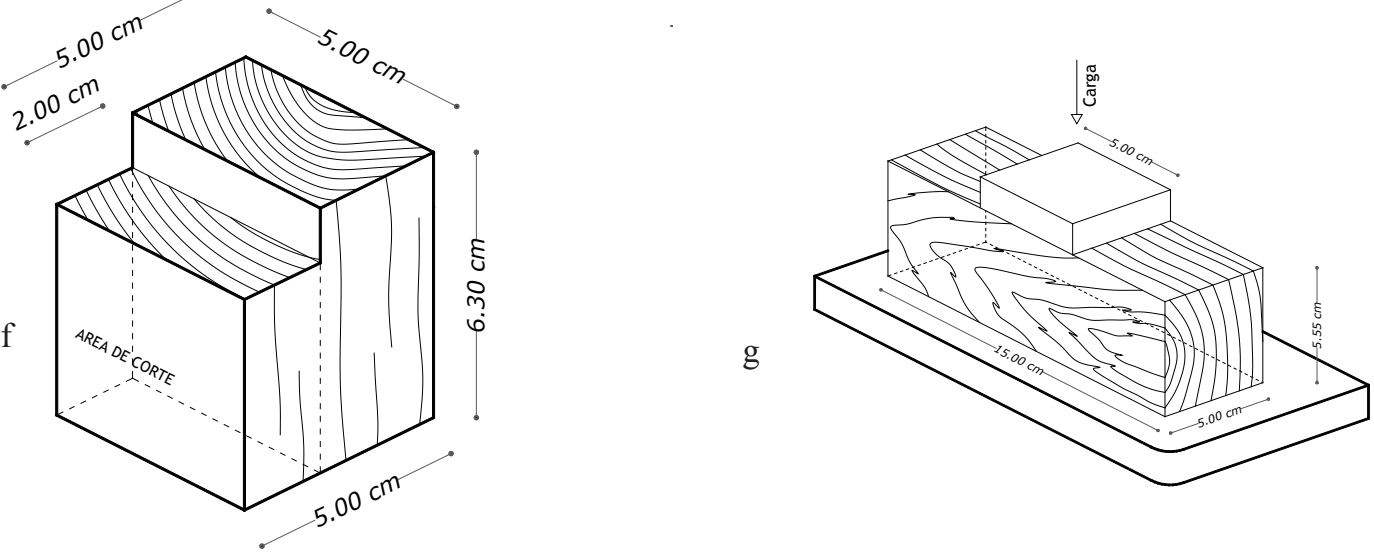

Figura a) Probeta para ensayos de las propiedades físicas de madera; b) Probeta y accesorios para el ensayo de flexión estática; c) Probeta y accesorios para el ensayo de compresion paralela a las fibras; d) Probeta y accesorios para el ensayo de dureza Brinnel en lados y extremos; e) Probeta y accesorios para el ensayo de extracción de clavos en lados y extremos; f) Probeta para el ensayo de corte paralelo a las fibras en la cara radial; g) Probeta y accesorios para el ensayo de compresión perpendicular a las fibras. 